\title{
Development and Validation of a Computational Fluid Dynamics (CFD) Solver for Droplet-Fibre Systems
}

\author{
$\underline{\text { R. Mead-Hunter }}^{\text {a }}$, B.J. Mullins ${ }^{\text {a }}{ }^{\mathrm{b}}$ A.J.C King ${ }^{\mathrm{a}}$ \\ ${ }^{a}$ Fluid Dynamics Research Group, Curtin University, GPO BOX U1987, Perth, Western Australia, 6845 \\ ${ }^{\mathrm{b}}$ School of Public Health, Curtin University, GPO BOX U1987, Perth, Western Australia, 6845 \\ Email: $\underline{\text { R.Mead-Hunter@curtin.edu.au }}$
}

\begin{abstract}
:
Droplet-fibre interactions are found in many natural and anthropogenic systems. A common industrial example is fibrous filtration - used to capture liquid (e.g. oil) mists. The filters used consist mostly of highly porous arrays of randomly layered fibres. Given the random (complex) nature of these filters, the existing models describing their behaviour are mainly empirical in nature and thus only applicable over a narrow range of parameters and operating conditions. Therefore simulation of these filters using computational fluid dynamics offers a viable alternative to the existing models.

In this work we will detail the development of a solver that couples the Lagrangian tracking of particles with a volume-of-fluid (VOF) solver. This solver is built on the existing open-source OpenFOAM CFD libraries, which have been modified to allow the physically accurate modelling of small particles. The solver also models the collection of these particles, where there is a transition from the discrete treatment (as in the Lagrangian tracking of the particles) to the volume-of-fluid treatment.

The solver allows the simulation of the motion of small liquid droplets, the capture of these droplets by filter fibres, the coalescence of these captured droplets, into films and the subsequent break up of these films into droplet arrays by Plateau-Rayleigh instability. Also simulated is the movement of these coalesced droplets within the filter, leading to the drainage of oil from the filter.

A validation of the fundamental physical mechanisms in the filter was performed, by comparing the simulated conformation of liquid droplets and films on the fibre to Plateau-Rayleigh instability theory. The model showed general agreement with both theory and observations. The simulated capture efficiency was also compared to capture efficiencies predicted by the single fibre efficiency (SFE) theory. A good agreement between the two was found.
\end{abstract}

Keywords: Droplet, fibre, computational fluid dynamics. 


\section{INTRODUCTION}

The most common method for the treatment of oil-mists is fibrous filtration. However, despite the prevalence of such technology in industry, relatively little in understood about the mechanisms of their behaviour. Given their complex nature and the many variables involved (fibre diameter, packing density, fibre material etc) accurate modelling of filter behaviour is difficult. The models currently available are predominantly empirically derived and apply only to specific cases. Instead of trying to model the filter using empirical equations then, it is perhaps more appropriate to simulate their behaviour. In order to do this, though, there will need to be a CFD solver capable of simulating droplet-fibre systems. Such a solver is presented here.

The capture efficiency of fibrous filters can be determined using a series of semi-empirical equations known as the single fibre efficiency (SFE) theory, where there are equations for the capture of particles by each of the capture mechanisms in play. In terms of fibrous filters handling liquid aerosols, the SFE equations used are for capture by interception, impaction, diffusion and the interaction between diffusion and interception.

Particle capture efficiency via interception may be found using,

$\eta_{R}=\frac{1-\alpha}{K u} \frac{N_{R}^{2}}{1+N_{R}}$

[Lee and Liu, 1982], where $\alpha$ is the packing density, $N_{R}$ is the ratio of droplet diameter to fibre diameter and $K u$ is the Kuwabara hydrodynamic function. The single fibre efficiency due to impaction, $\eta_{I}$, may be determined from the following equation; developed by Stechkina et al. [1969],

$\eta_{I}=\frac{S_{t}}{4 K u^{2}}\left[\left(29.6-28 \alpha^{0.62}\right) N_{R}^{2}-27 N_{R}^{2.8}\right]$

Where $S t$ is the Stokes number which is found using,

$S_{t}=\frac{\rho_{p} d_{p}^{2} C_{c} u^{\prime}}{18 \mu_{g} d_{f}}$,

where $\rho_{p}$ is the particle density, $d p$ the particle diameter, $u$ ' is the interstitial velocity and $C c$ is the Cunningham correction factor.

The single fibre efficiency by diffusion, $\eta_{D}$, can be described by the expression,

$\eta_{D}=2.7 P e^{-\frac{2}{3}}$

[Cheng et al., 1990], where $P e$ is the Peclet number, and the interaction between diffusion and interception may be described by,

$\eta_{D R}=\frac{1.24 N_{R}^{\frac{2}{3}}}{(K u P e)^{\frac{1}{2}}}$.

[Hinds, 1999]. The single fibre efficiency is then determined from;

$\eta_{F}=1-\left(1-\eta_{R}\right)\left(1-\eta_{I}\right)\left(1-\eta_{D}\right)\left(1-\eta_{D R}\right)$.

With the efficiency of the filter determined using,

$\eta_{T}=1-\exp \left(\frac{-4 \alpha \eta_{F} Z}{\pi d_{f}}\right)$ 
where $Z$ is the filter thickness.

The SFE theory has been found to be very accurate in determining the capture efficiency of filters and as such provides us with a way to validate the simulated particle capture using our CFD solver.

Once collected, the oil droplets spread along the fibre and form a thin film (provided the fibre is wettable), this film though is unstable and will be broken up by the well known phenomena of Plateau-Rayleigh instability.

Mullins and Kasper [2006] have shown that on a cylindrical element (such as a fibre) there is a limit on the film thickness, $h t$, of:

$h_{t}=r(\sqrt{2}-1)$.

This then provides a means of validating the simulated behaviour of collected liquid, as modelled by the solver. With regards to fibrous filtration it should also be remembered that the onset of Plateau-Rayleigh instability will also be influenced by the presence of overlapping and intersecting fibres as well as air flow through the filter. This means that the maximum film thickness may in fact be less than indicated by Equation (8).

The Open Field Operation And Manipulation (OpenFOAM) CFD package was selected for this work, as it is free and open-source and therefore easily modifiable/editable. It comes with a robust Lagrangian tracking algorithm, which is essential for this work. Additionally, the user is less restrained in the use of different packages and solvers and can couple different solvers such that work can be done without the need for it to be anticipated by the software developers. OpenFOAM is also advantageous over commercial packages as it allows the user into the code so that, they can see exactly what is being implemented and how.

\section{SOLVER DEVELOPMENT}

\subsection{Particles}

Each particle is individually tracked through the flow field until it is collected or exits the geometry. The particle tracking is done within a Lagrangian frame of reference, with the integration of particle position and velocity done explicitly at least once per time-step [King and Mullins, 2011]. In order to accurately model the motion of particles, the forces acting on the particles had to be incorporated.

The buoyancy forces, $F_{b}$, are described by,

$F_{t}=\frac{\pi d_{p}^{3}}{6}\left(\rho_{p}-\rho_{g}\right) g$

where $\rho_{g}$ in these simulations is simply the density of air, $\rho_{p}$ is the density of the particle, which will be the density of the oil (i.e. the particles are really liquid droplets) and $d_{p}$ is the diameter of the particle.

The drag forces, $F_{d}$, are described by,

$F_{d}=\frac{18 \mu}{\rho_{p} d_{p}^{2}} \frac{C_{D}}{24} R e$

where $C_{D}$ is the drag coefficient, determined using the model of Haider and Levenspiel [1989] with,

$C_{D}=\frac{24}{R e}\left(1+0.1806 R e^{0.6459}\right)+\frac{0.4251}{1+\frac{6880.95}{R e}}$.

For small particles (like the ones encountered in oil-mists) thermal diffusion (Brownian motion) is significant. The force then acting on the particle due to thermal diffusion, $F_{t}$ is described by,

$F_{t}=\mathbf{G}\left(\frac{\pi S_{0}}{\Delta t}\right)^{\frac{1}{2}}$ 
where $\boldsymbol{G}$ is a vector whose components are independent zero-mean, unit variance Gaussian random numbers [Ounis et al., 1990].

\subsection{Particle Tracking}

One of the reasons for selecting the OpenFOAM CFD package was that it contains a robust particle tracking algorithm. The algorithm itself is presented and discussed in detail by Macpherson et al. [2009] in their work.

\subsection{Fluids}

The volume-of-fluid (VOF) method is utilised for the simulation of liquid in the solver. In OpenFOAM the VOF method is incorporated into the standard solver, interFoam. The solver that is being developed is result of the coupling the particle tracking solver with interFoam. This solver is known as twoPhaseOilParticleFoam and is used for the simulation of droplet fibre systems.

In addition to the momentum and continuity equations the VOF method involves a transport equation for the volume fraction of on phase (the oil in the case of coalescing filters), which includes interface compression;

$$
\frac{\delta \alpha}{\delta t}+\nabla \bullet\left(\mathbf{U} \alpha_{l}\right)+\nabla \bullet\left[U_{r} \alpha_{l}\left(1-\alpha_{l}\right)\right]=S_{\alpha},
$$

with $U_{r}$ being the compression velocity, $\alpha_{l}$ the fluid volume fraction and $S_{\alpha}$ a phase fraction source term. Note that the compression velocity corresponds to the difference between the liquid and gas velocities and is not related to compressible flows [Berberovic et al., 2009].

The solver twoPhaseOilParticleFoam has been developed as a three-dimensional unsteady solver that utilises the Multidimensional Universal Limiter with Explicit Solution (MULES) algorithm for the transport equation (Equation 13) and the Pressure Implicit with Splitting of Operators (PISO) algorithm to solve for the coupled pressure-velocity fields [King and Mullins, 2011].

\subsection{Particle Collision}

A simple algorithm is implemented to check for collision between particles. For each cell, a list of particles and their positions is generated. The positions of all the particles in the cell (and those in the neighbouring cells) are then compared and any particles that are closer than the sum of their individual radii are considered to have collided. Particles that have collided are deleted and replaced with a new particle.

\subsection{Particle Collection}

A particle is collected if its trajectory passes within one particle radius of a boundary or intersects the boundary. Given the use of a cyclic boundary condition, the only areas that particles may be collected is at the outlet or on the fibres.

The collection of particles by oil, (which has been previously collected on the fibres), is determined by considering the volume fraction of the cell. If the fluid volume fraction, $\alpha_{l}$, in a cell is greater than 0.5 , then any particle in that cell is considered collected by the oil. Where the value of 0.5 was chosen as a basis upon which to identify cells containing the liquid-gas (oil-air) interface.

Any particle collected by the oil is marked as deleted. To preserve continuity, the mass of the collected particle must be incorporated into the transport equation (Equation 13). This is achieved by adjusting the volume fraction of the cell to include the volume of the collected droplet. As the particle is an oil droplet, which is collected by the same oil which is already in the cell, the densities are obviously the same and therefore by conserving volume the mass is being conserved also. The adjusted volume fraction of the cell is then,

$\alpha_{l}=\frac{\pi d_{p}^{3}}{6} \frac{1}{V_{\text {cell }}}$, 
where $V_{\text {cell }}$ is the volume of the cell [King and Mullins, 2011].

Additionally, the momentum must also be conserved so a source term is added to momentum equation, to account for the momentum of the collected (deleted) particle. The momentum source term is then,

$S_{m}=\rho_{p} u_{p}$,

where $u_{p}$ is the velocity of the particle, when it was collected.

\subsection{Conversion from Particles to a Volume of Fluid}

In order for the behaviour of a coalescing filter to be simulated, the solver must be able to transition form the tracking of individual particles to a volume of fluid treatment without relying on a fluid volume already in place. This will always be case when a clean filter is considered at the start of a simulation. The transition from Lagrangian tracking of individual particles to a VOF method is achieved in twoPhaseOilParticleFoam by considering the ratio of particle volume to cell volume. If the value of the ratio reaches a value of 1 (i.e. the cell is full of fluid) then the particles are deleted and the adjusted volume fraction and momentum source term are calculated and added to the transport and momentum equations respectively.

\section{RESULTS AND DISCUSSION}

\subsection{Examination of Particle Capture}

The capture of particles using the solver dustParticleFoam was examined by comparing the results of simulations with the efficiencies predicted using the single fibre efficiency theory. To achieve this a geometry has to be prepared, for which the single fibre efficiency is known to be accurate. For this purpose a regular mesh geometry was used consisting of four fibres forming a grid pattern. As this is a geometry for which the SFE is very accurate.

Initial simulations conducted showed that particle capture was dependent on the size of the mesh used. This effect was explored by altering the size of the mesh in the blockMeshDict file. A series of meshes were prepared, all using the grid pattern geometry, with cell sizes ranging from $5 \mu \mathrm{m}$ to $1.667 \mu \mathrm{m}$. Simulations were then run on each mesh using particle diameters of 50 to $1000 \mathrm{~nm}$. The simulated capture efficiencies (simply the number of particles that are collected by the fibres divided by the total number injected) are shown in Figure 1, with the SFE theory predicted efficiencies.

As shown in Figure 1, the coarser meshes tend to over predict capture efficiency for all particle diameters, but form the same shaped curve as the SFE theory predicted values. Essentially the simulated results appear as though they could be described by a translation of the SFE theory predicted curve. As the mesh used becomes finer the values for the small particles (where diffusion is the main capture mechanism in place) approach the predicted values. For particles less than $500 \mathrm{~nm}$ in diameter the agreement between the simulated and predicted values is very good for the finest mesh. However, the simulated capture efficiencies for the larger particles (where interception is dominant) are less than the predicted efficiencies when finer meshes are used.

The mesh dependency has an additional effect on the larger particles. Whereas for particles of diameters smaller than $600 \mathrm{~nm}$ the simulated efficiency approaches the SFE predicted efficiency, the simulated efficiencies for larger particles do not and the shape of the curve deviates from the shape of the typical capture efficiency curve. This is due to an issue with the way the particles are treated. Particles are tracked as a point mass, located at the centre of the particle; therefore the centre of the particle may be located in one cell, but part of the outer surface of the particle could be in a neighbouring cell. As the cell size is reduced (which is the obvious result of using a finer mesh) the size of the larger particles become closer to the size of the cell. Hence it becomes increasingly likely that a particle may be located in two or three (potentially more) different cells. This is not an issue for the tracking algorithm, but it can make it difficult for the solver to determine that a particle has been (should be) captured.

The reason for the deviation in the shape of the efficiency curve is then due to a particle passing a fibre, close enough to be captured (i.e. within 1 particle radius) where the solver does not recognise the particle as being captured as the centre of the particle is in a cell which is not immediately adjacent to the fibre. 


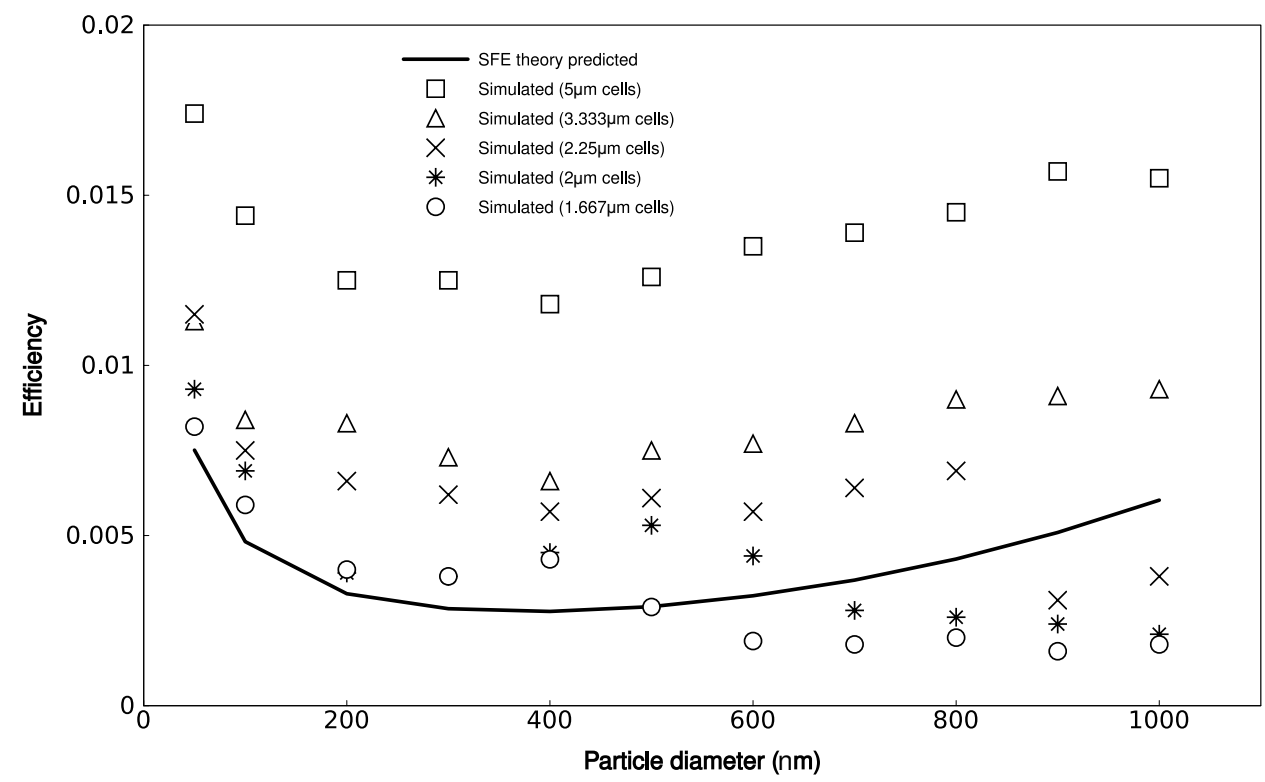

Figure 1: Simulated efficiencies using different mesh sizes, compared to SFE theory predicted efficiencies

This problem may be avoided by using a coarser mesh, which has cell sizes that are larger than the largest particle being simulated. This would allow the efficiency plot to maintain the correct shape though would mean that capture efficiency was over-predicted by a factor of approximately 2.3. Alternatively if particles smaller than $600 \mathrm{~nm}$ in diameter are used then use of a finer mesh will give simulated efficiencies that agree well with the SFE theory predicted efficiencies.

\subsection{Examination of the Plateau-Rayleigh Instability}

The development of the solver has dealt with the Lagrangian tracking of particles, and has been examined by comparison to the SFE theory. So in this state the solver is able to handle the tracking of discrete particles and the collection of these particles by surfaces as well as volumes of fluid (i.e. large droplets). However, in order to model the behaviour of a coalescing filter, the solver needs to accurately model the collection of discrete particles (oil droplets) by fibres, the spreading of these droplets over the fibre surface, film formation and the subsequent break up of the film by Plateau-Rayleigh instability.

Simulations were run on a geometry containing a single fibre; where a fluid column was placed around the fibre by using the funkySetFields utility.

In order to test the modelling of the Plateau-Rayleigh instability the thickness of the film was varied each simulation to see if the break up of the film would occur. The maximum possible film thickness for a given system can be determined using Equation 8. Therefore by simulating film thicknesses either side of this maximum value the effectiveness of OpenFOAM in simulating the Plateau-Rayleigh instability can be determined.

According to Equation 8 the thickest film that could exist on a 10 micron diameter fibre is $2.07 \mu \mathrm{m}$. Therefore film thicknesses from 1 to $3 \mu \mathrm{m}$ were examined, with the maximum stable film thickness found to be $1.4 \mu \mathrm{m}$. The simulations were run in the presence of a flow field, with an inlet velocity of 0.1 $\mathrm{m} / \mathrm{s}$, as in the simulations of droplet-fibre behaviour in filter will be in the presence of flow.

Therefore the simulated break-up occurs at a lower value of $h_{t}$ than predicted by Equation 8 . This however is permissible, as the value obtained from Equation 8 represents a maximum allowable film thickness, and the break-up of films is expected to occur earlier (i.e. at a smaller film thickness) in the presence of flow. Therefore it is clear that the solver will not allow a physically unrealisable film to exist 
on the fibre.

To completely demonstrate the implementation of Plateau-Rayleigh instability a film that is just below the maximum thickness was examined while collecting particles to see if the film will grow in thickness and subsequently break-up due to the collection of particles. Also the same simulation was run starting with a clean fibre and allowing it to collect droplets until the formation of axisymmetrical coalesced droplets occurred.

\section{CONCLUSION}

A CFD Solver has been presented which can be used to simulate droplet-fibre systems. It has been found that the solver can realistically simulate the transition of a liquid film into a an array of droplets, via the Plateau-Rayleigh instability and can also simulate the capture of particles. It is anticipated that the deviation form the SFE theory predicted values found for the larger particles may be removed by implementing an algorithm that searches neighbouring cells for boundaries.

\section{ACKNOWLEDGEMENTS}

This work was supported by an Australian Research Council Linkage Grant (LP0883877).

\section{REFERENCES}

Berberovic, E., N. van Hinsberg, S. Jakirlic, I. Roisman, and C. Tropea (2009). Drop impacr onto a liquid layer of finite thickness: Dynamics of cavity evolution. Physical Review E 79(3), 036306-1-03630615.

Cheng, Y., Y. Yamada, and H. Yeh (1990). Diffusion deposition on model fibrous filters with intermediate porosity. Aerosol Science and Technology 12(2), 286-299.

Haider, A. and O. Levenspiel (1989). Drag coefficient and terminal velocity of spherical and nonspherical particles. Powder Technology 58(1), 63-70.

Hinds, W. (1999). Aerosol Technology - Properties, Behaviour and Measurement of Airborne Partices (2 ed.). New York: John Wiley \& Sons.

King, A. and B. J. Mullins (2011). A new computational fluid dynamics solver to simulate capture, coalesecnce and drainage in coalescing filter media. American Filtration and Separations Society, 24th Annual Conference, Louisville, USA.

Lee, K. W. and B. Y. H. Liu (1982). Theoretical study of aerosol filtration by fibrous filters. Aerosol Science and Technology 1(2), 147 - 161.

Macpherson, G., N. Nordin, and H. Weller (2009). Particle tracking in unstructured, arbitrary polyhedral meshes for use in cfd and molecular dynamics. Communications in Numerical Methods in Engineering 25(3), 263-273.

Mullins, B. J. and G. Kasper (2006). Comment on: Clogging of fibrous filters by liquid aerosol particles: Experimental and phenomenological modelling study by frising et al. Chemical Engineering Science 61(18), $6223-6227$.

Ounis, H., G. Ahmadi, and McLaughlin (1990). Brownian diffusion of submicrometer particles in the viscous sublayer. Journal of Colloid and Interface Science 143(1), 266-277.

Stechkina, I. B., A. A. Kirsch, and A. Fuchs (1969). Studies on fibrous aerosol filters - iv calculation of aerosol deposition in model filters in the range of maximum penetration. Annals of Occupational Hygiene 12(-), 1-8. 